\title{
Analysis and Design of an Array of Two Differential Oscillators Coupled Through a Resistive Network
}

\author{
Mihaela Izabela Ionita \\ Electrical Engineering Faculty \\ University Politehnica of Bucharest \\ Bucharest, Romania \\ LAII \\ University of Poitiers \\ Angouleme, France \\ mihaelaizabela@yahoo.com
}

\author{
David Cordeau, Jean Marie Paillot, Mihai Iordache* \\ LAII \\ University of Poitiers \\ Angouleme, France \\ * Electrical Engineering Faculty \\ University Politehnica of Bucharest \\ Bucharest, Romania \\ david.cordeau@univ-poitiers.fr; jean.marie.paillot@univ- \\ poitiers.fr; iordache@elth.pub.ro
}

\begin{abstract}
This paper considers the analysis and the design of an array of two NMOS differential oscillators coupled through a resistor. A new writing of the nonlinear equations proposed by $R$. York to describe the oscillators' locked states but limited for the specific case of a resistive coupling is presented. The new system permits the calculation of the free-running frequencies of the oscillators when a specific phase shift is desired. This has led to the modeling of the two coupled NMOS differential oscillators as two coupled differential Van der Pol oscillators, with a resistive coupling network. A good agreement between the circuit, the model and the theory was found, giving some design considerations for a network of two differential oscillators coupled through one resistor.
\end{abstract}

Keywords: $R L C$ differential oscillator, resistive coupling network, differential Van der Pol oscillator, synchronization.

\section{INTRODUCTION}

Arrays of coupled oscillators are used to produce higher powers at millimeter-wave frequencies with better efficiency than is possible with conventional power-combining techniques. Another application is the beam steering of antenna arrays. In this case, the radiation pattern of a phased antenna array is steered in a particular direction through a constant phase progression in the oscillator chain which is obtained by detuning the free-running frequencies of the outermost oscillators in the array [1]. Furthermore, it is shown that the resulting inter-stage phase shift is independent on the number of oscillators in the array [2-4].

In his previous works, R. York made use of simple singleended Van der Pol oscillators to model coupled microwave oscillators. Based on a generalization of Kurokawa's method [5], he was able to derive the equations for the amplitude and phase dynamics of $N$ oscillators coupled through many types of circuits [6,7]. Unfortunately, it is shown in [2] that the theoretical limit of the phase shift that can be obtained by slightly detuning the end elements of the array by equal amounts but in opposite directions is only $\pm 90^{\circ}$. Thus, it seems to be interesting to study and analyze the behavior of an array of coupled differential oscillators since, in this case, the theoretical limit of the phase shift is within $360^{\circ}$ due to the differential operation of the array. In this case, with the use of such an array, a continuously controlled $360^{\circ}$ phase shifting range can be achieved leading to an efficient beam-scanning architecture for example. Furthermore, differential oscillators are widely used in high-frequency circuit design due to their relatively good phase noise performances and ease of integration. Moreover, the use of a resistive coupling network instead of a resonant one can lead to a substantial save in chip area.

Due to these considerations, the aim of this paper is to present the analysis and the design of an array of two differential NMOS oscillators coupled through a resistive network. To do so, the equations developed by R. York giving the dynamics for two single-ended Van Der Pol oscillators coupled through a resonant network will be overviewed in subsection II-A. In subsection II-B, a new writing of these equations for the specific case of a resistive coupling will be presented. In section III, the study and the modeling of the two NMOS RLC differential oscillators coupled through a resistor will be described followed by the conclusion.

\section{THEORETICAL ANALYSIS}

A. Dynamics of two oscillators coupled through a resonnant circuit

The study of the dynamics of an array of two oscillators is done using a Van der Pol model. This model is made of a RLC resonator and a nonlinear conductance, which has the following expression:

$$
G_{N L}=-a_{1}+a_{3} V^{2}
$$

where $-a_{1}$ is the negative conductance necessary to start the oscillation and $a_{3} V^{2}$ is the nonlinear conductance which modelises the saturation phenomenon. 
In [7], R. York \& J. Lynch derived the equations for the amplitude and phase dynamics of two single-ended Van der Pol oscillators coupled through a RLC circuit, as follows :

$$
\begin{gathered}
\left(1-\lambda_{o} \varepsilon^{2}-A_{1}^{2}\right) A_{1}=-\lambda_{o} \varepsilon A_{2} \cos (\Delta \phi-\Phi) \\
\Delta \omega_{o 1}-\left(1-\lambda_{o} \varepsilon^{2} \frac{\omega_{a}}{\omega_{a c}}\right) \Delta \omega_{c}=-\lambda_{o} \varepsilon \omega_{a} \frac{A_{2}}{A_{1}} \sin (\Delta \phi-\Phi) \\
\left(1-\lambda_{o} \varepsilon^{2}-A_{2}^{2}\right) A_{2}=-\lambda_{o} \varepsilon A_{1} \cos (\Delta \phi+\Phi) \\
\Delta \omega_{o 2}-\left(1-\lambda_{o} \varepsilon^{2} \frac{\omega_{a}}{\omega_{a c}}\right) \Delta \omega_{c}=\lambda_{o} \varepsilon \omega_{a} \frac{A_{1}}{A_{2}} \sin (\Delta \phi+\Phi)
\end{gathered}
$$

where

$$
\varepsilon=\frac{1}{\sqrt{1+\left(\frac{\Delta \omega_{c}}{\omega_{a c}}\right)^{2}}}
$$

and

$$
\Phi=\tan ^{-1}\left(\frac{\Delta \omega_{c}}{\omega_{a c}}\right)
$$

are the coupling strength scale factor and coupling phase that result from frequency-dependent attenuation and phase delay through the coupling circuit and $|\Phi|<90^{\circ}$. The ability of these coupled oscillators to synchronize to a common frequency is affected by the following parameters [8]:

- $\lambda_{o}=\frac{1}{G_{0} R_{c}}$ : coupling constant, where $G_{0}$ is the firstorder term of Van der Pol nonlinear conductance

- $2 \omega_{a}=\frac{G_{0}}{C}:$ frequency bandwidth of the oscillators

- $2 \omega_{a c}=\frac{R_{c}}{L_{c}}$ : frequency bandwidth of the unloaded coupling circuit

- $\omega_{o 1}, \omega_{o 2}$ : free-running frequencies or tunings of oscillators 1 and 2 , respectively

- $\omega_{o c}$ : resonant frequency of the coupling circuit

Furthermore, let us note that in (1), $\omega_{o 1}$ and $\omega_{o 2}$, and the synchronization frequency of the system, $\omega$, are referred to the resonant frequency of the coupling circuit, $\omega_{o c}$, using the following substitutions:

$$
\Delta \omega_{o 1}=\omega_{o 1}-\omega_{o c} \quad \Delta \omega_{o 2}=\omega_{o 2}-\omega_{o c} \quad \Delta \omega_{c}=\omega-\omega_{o c}
$$

\section{B. New writing of the equations for the Broad-Band case}

The case when the coupling circuit is made of one resistor implies $\omega_{a c} \rightarrow \infty$, which means that $\varepsilon=1$ and $\Phi=0$ according to (1). In these conditions, the new equations describing the locked states can be written as:

$$
\begin{gathered}
\left(1-\lambda_{o}-A_{1}^{2}\right) A_{1}=-\lambda_{o} A_{2} \cos (\Delta \phi) \\
\omega_{o 1}=\omega-\lambda_{o} \omega_{a} \frac{A_{2}}{A_{1}} \sin (\Delta \phi) \\
\left(1-\lambda_{o}-A_{2}^{2}\right) A_{2}=-\lambda_{o} A_{1} \cos (\Delta \phi) \\
\omega_{o 2}=\omega+\lambda_{o} \omega_{a} \frac{A_{1}}{A_{2}} \sin (\Delta \phi)
\end{gathered}
$$

If one want to obtain the maximum phase shift, the synchronization frequency must be located between the two oscillators free-running frequencies as explained in [7] and called the line of "equally spaced tunings", so that :

$$
\omega=\frac{\omega_{o 1}+\omega_{o 2}}{2}
$$

Then, according to (2), this implies $A_{1}=A_{2}$ and the frequency difference between the two free-running frequencies of oscillators 1 and 2 can be expressed as follows:

$$
\Delta \omega_{o}=2 \lambda_{o} \omega_{a} \sin (\Delta \phi)
$$

with $\Delta \omega_{o}=\omega_{o 2}-\omega_{o 1}$ as mentioned previously.

As a consequence, one can note that (3) can be used to find the tunings $\left(\omega_{o 1}\right.$ and $\left.\omega_{o 2}\right)$ of the two oscillators for a given phase shift $\Delta \phi$. Nevertheless, to do so, the parameters $\lambda_{o}, \omega_{a}$ and thus the Van Der Pol model of the oscillator must be known.

\section{STUDY AND MODELING OF TWO COUPLED DIFFERENTIAL OSCILLATORS}

\section{A. The RLC Differential Oscillator}

In this paper, the oscillator schematic used in simulations is depicted in Figure 1. It is based on the well-known crosscoupled NMOS differential topology using a $0.35 \mu \mathrm{m}$ BiCMOS SiGe process. The cross connected NMOS differential pair provides the negative resistance to compensate for the tank losses. The tail current source is a simple NMOS current mirror and draws $28 \mathrm{~mA}$ with a ratio of 14 in order to reduce the power consumption. The frequency of oscillation is determined by the $L C$ tank at the drains, so that the oscillation frequency is close to $6 \mathrm{GHz}$. In these conditions, the inductance value, $L$, is close to $0.8 \mathrm{nH}$ and the capacitor value, $C$, is close to $0.88 \mathrm{pF}$. The resistor value, $R$, is equal to $100 \Omega$ so that the quality factor of the tank is equal to 3.3. A tail capacitor $C_{T}$ is used to attenuate both the high-frequency noise component of the tail current and the voltage variations on the tail node. This latter effect results in more symmetric waveforms and smaller harmonic distortion in LC-VCO outputs [9].

To ensure proper start-up of the oscillator, the transconductance of the NMOS transistor should be greater than $\frac{1}{R}$. In these conditions, the sizes of NMOS transistors $T_{1}$ and $T_{2}$ are identical and chosen to be $\left(\frac{W}{L}\right)=\frac{70 \mu \mathrm{m}}{0.35 \mu \mathrm{m}}$. 


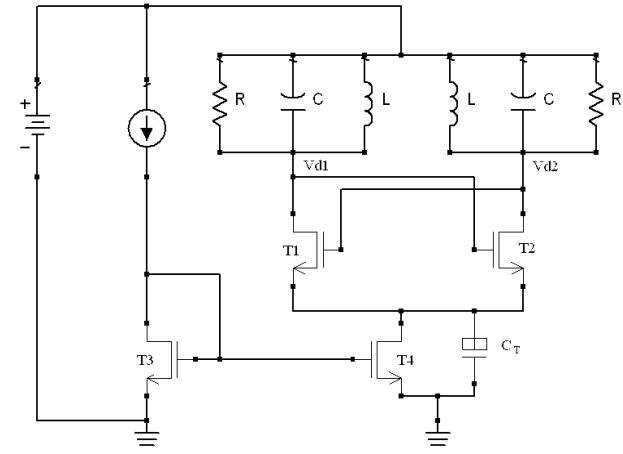

Figure 1. RLC NMOS differential oscillator schematic

\section{B. Modeling of the array of two differential oscillators using} a differential Van Der Pol model

In order to represent the oscillator of Fig. 1 by a negative resistance in parallel with an RLC resonator, Agilent's ADS software was used. From ADS simulation results for the differential oscillator at the required synchronization frequency, it is then possible to extract the parallel RLC circuit that models the resonator, as well as the parameters $a_{1}$ and $a_{3}$ of the Van der Pol equation capable of reproducing the behaviour of the oscillator's active part. In these conditions, the differential oscillator represented in Fig. 1 can be reduced into a new Van der Pol differential oscillator with a RLC resonator as shown in Fig. 2. Thus, for an oscillation frequency close to 6 $\mathrm{GHz}, R$ is equal to $200 \Omega$, the inductance value, $L$, is close to $1.6 \mathrm{nH}$ and the capacitor value, $C$, close to $0.44 \mathrm{pF}$.

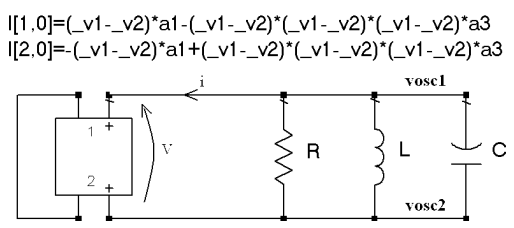

Figure 2. The differential Van der Pol oscillator model with $i=-a_{1} v+a_{3} v^{3}$

Fig. 3 shows the waveforms of the differential output voltages for the RLC NMOS differential oscillator of Fig.1 as well as those of the differential Van der Pol oscillator model at a frequency of $5.970 \mathrm{GHz}$. As can be seen in this figure, a very good agreement is found between the behavior of the model and the circuit.

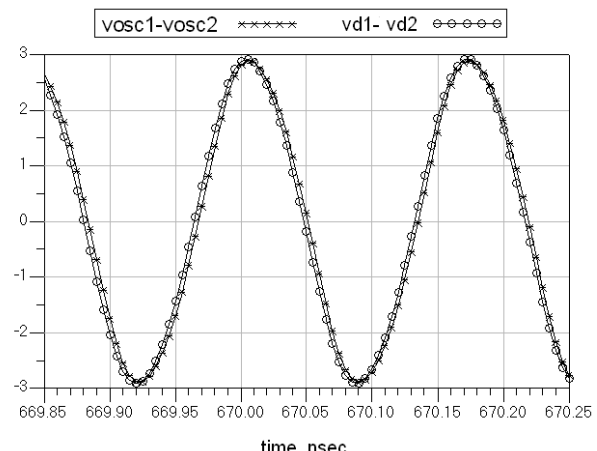

Figure 3. Comparison between the output voltages of the differential oscillator and the differential Van der Pol oscillator model
Let us now consider the two identical differential Van der Pol oscillators coupled through one resistor of $200 \Omega$, like in Fig. 4. In this case, the value of the coupling resistor on each path is $R_{c} / 2$ to match well with the theory based on the use of two single-ended Van Der Pol oscillators. Now using (3), the range of frequencies over which these two coupled oscillators lock can be obtained. For instance, if the desired phase shift for the targeted application is $42^{\circ}$, the free-running frequencies of the two oscillators are $f_{o l}=5.67 \mathrm{GHz}$ and $f_{o 2}=6.27 \mathrm{GHz}$ for $\lambda_{o}=0.5$ and $\omega_{a}=5.68 .10^{9} \mathrm{rad} / \mathrm{s}$.

In these conditions, this coupled system simulated with ADS leads to four sinusoidal waves at a synchronization frequency $f_{s}$ of $5.96 \mathrm{GHz}$ with a phase shift equal to $41.6^{\circ}$ as shown in Fig. 5.

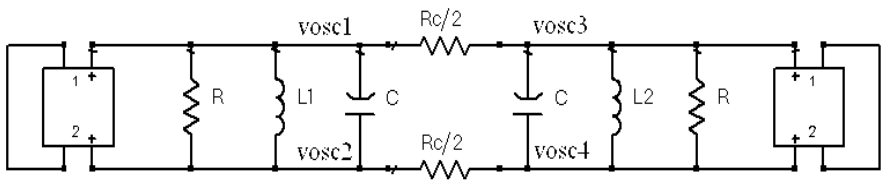

Figure 4. The two differential Van der Pol osillators coupled through a resistor

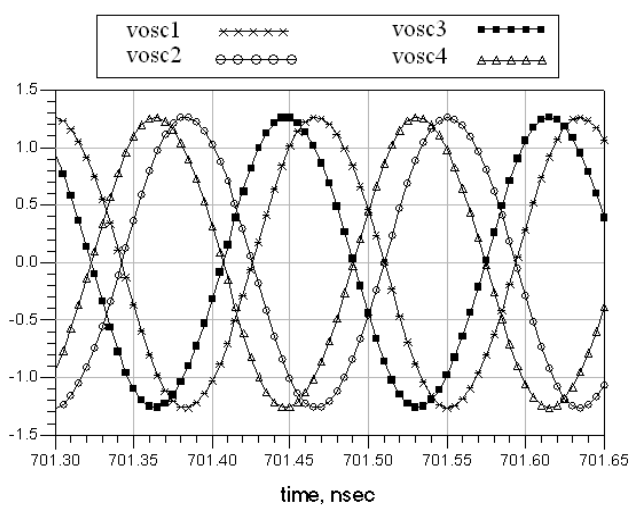

Figure 5. Waveforms of the output voltages for the coupled differential Van der Pol oscillators for $\Delta \phi=41.6^{\circ}$

One can note that a good agreement was found between the theoretical prediction of the phase shift and the simulation results of the differential Van Der Pol model developed.

\section{The Two coupled RLC NMOS differential oscillators}

In order to design the array made of the two coupled differential oscillators, the theory for the coupled differential Van der Pol oscillators was used. Thus, under the same simulation conditions used for two differential Van der Pol oscillators, the two differential oscillators were coupled through a resistor of $200 \Omega$, as shown in Fig. 6. In these conditions, Fig. 7 shows the differential output voltages waveforms of the two differential oscillators of Fig. 6 as well as those of the differential Van Der Pol model. In this case, the two coupled differential oscillators are synchronized at 5.98 $\mathrm{GHz}$ with a phase shift $\Delta \phi=41.3^{\circ}$.

As a consequence, one can see that a very good agreement is found between the behavior of the model and the circuit. 


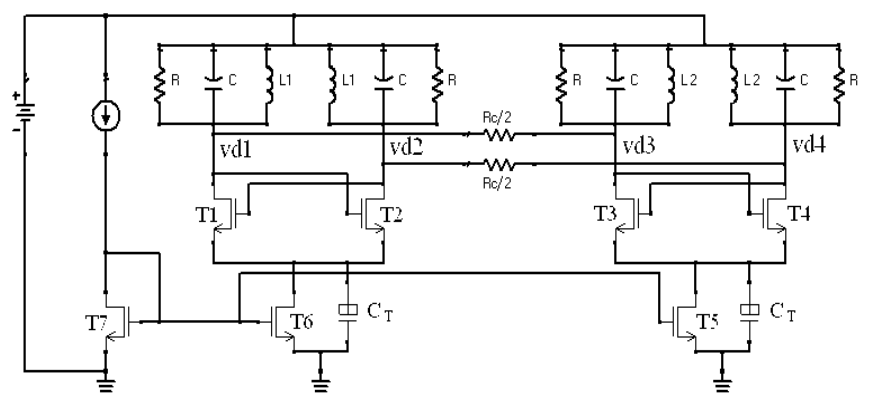

Figure 6. Two differential oscillators coupled through a resistor

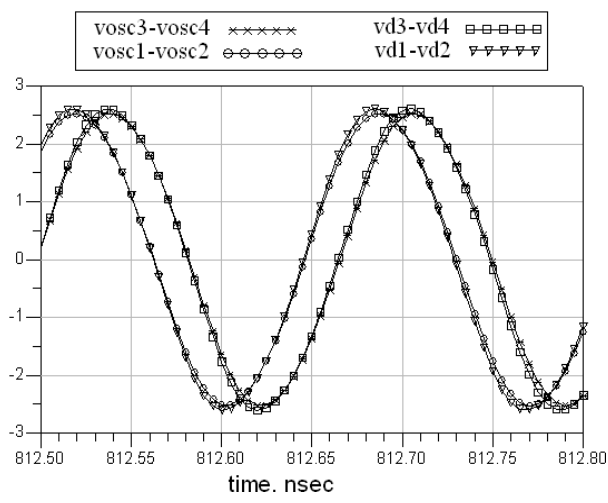

Figure 7. Comparison between the output voltages of the coupled differential oscillators and the coupled differential Van der Pol oscillators, when $\Delta \phi=41.3^{\circ}$

Now, in order to show the ability of the system to achieve the desired phase shift and to find the range of frequencies over which these two coupled oscillators can lock, we changed the free-running frequencies of the both pairs of oscillators so that the synchronization frequency will remain the same. Table I shows a comparison between the synchronization frequency and the phase shift obtained with the coupled NMOS differential oscillators, the coupled differential Van der Pol oscillators and by using the theory.

TABLE I. COMPARISON OF THE OBTAINED RESULTS FOR THE SYNCHRONIZATION FREQUENCY AND THE PHASE SHIFT

\begin{tabular}{|c|c|c|c|c|c|c|c|}
\hline \multirow[t]{2}{*}{$\begin{array}{c}f_{o I} \\
G H z\end{array}$} & \multirow[t]{2}{*}{$\begin{array}{c}f_{o 2} \\
G H z\end{array}$} & \multicolumn{3}{|c|}{$\begin{array}{c}f s \\
G H z\end{array}$} & \multicolumn{3}{|c|}{$\Delta \phi$} \\
\hline & & $\begin{array}{l}\text { diff. } \\
\text { osc. }\end{array}$ & $\begin{array}{l}\text { VDP } \\
\text { Osc. }\end{array}$ & theory & $\begin{array}{l}\text { diff. } \\
\text { osc. }\end{array}$ & $\begin{array}{l}\text { VDP } \\
\text { Osc. }\end{array}$ & theory \\
\hline 5.97 & 5.97 & 5.96 & 5.94 & 5.97 & $0^{\circ}$ & $0^{\circ}$ & $0^{\circ}$ \\
\hline 6.03 & 5.91 & 5.96 & 5.94 & 5.97 & $7.1^{\circ}$ & $7.2^{\circ}$ & $7.6^{\circ}$ \\
\hline 6.09 & 5.85 & 5.98 & 5.96 & 5.97 & $15.7^{\circ}$ & $15.9^{\circ}$ & $15.5^{\circ}$ \\
\hline 6.15 & 5.79 & 5.98 & 5.96 & 5.97 & $24.6^{\circ}$ & $24.8^{\circ}$ & $23.6^{\circ}$ \\
\hline 6.21 & 5.73 & 5.98 & 5.96 & 5.97 & $31.2^{\circ}$ & $31.4^{\circ}$ & $32.3^{\circ}$ \\
\hline 6.27 & 5.67 & 5.98 & 5.96 & 5.97 & $41.3^{\circ}$ & $41.6^{\circ}$ & $42.0^{\circ}$ \\
\hline 6.33 & 5.61 & 5.99 & 5.96 & 5.97 & $53.4^{\circ}$ & $53.8^{\circ}$ & $53.4^{\circ}$ \\
\hline 6.39 & 5.55 & 5.99 & 5.96 & 5.97 & $68.7^{\circ}$ & $69.4^{\circ}$ & $69.5^{\circ}$ \\
\hline 6.42 & 5.53 & 5.99 & 5.96 & 5.97 & $81.0^{\circ}$ & $82.0^{\circ}$ & $83.1^{\circ}$ \\
\hline
\end{tabular}

One can see that a good agreement was found between these results. The maximum value of the phase shift is obtained for $f_{o 1}=6.42 \mathrm{GHz}$ and $f_{o 2}=5.53 \mathrm{GHz}$. Above this values of the free-running frequencies, the oscillators are not able to synchronize anymore.

Thus, these results show that it is possible to adjust, with a relatively high accuracy, the free-running frequencies of the two differential NMOS oscillators required to achieve the desired phase shift.

\section{CONCLUSIONS}

This paper was focused on the analysis and the design of an array of two differential NMOS oscillators coupled through a resistive network. Starting from the system of nonlinear equations presented in [7] by R. York, a simpler system reduced to the case of a resistive coupling network and allowing to determine the free-running frequencies of the two oscillators required to achieve the desired phase shift was developed. After that, the modeling of the chosen NMOS RLC differential oscillators as one differential Van der Pol oscillator was performed. Thus, under the same simulation conditions used for two coupled differential Van der Pol oscillators, the two differential NMOS oscillators were coupled through a resistive network. A good agreement between the results obtained for the coupled NMOS differential oscillators, the coupled differential Van der Pol oscillators and the theory was found showing the potential of this new oscillator array architecture.

\section{REFERENCES}

[1] P. Liao and R. A. York, "A new phase-shifterless beam-scanning technique using arrays of coupled oscillators", IEEE Transactions on Microwave Theory and Techniques, vol. 41, $\mathrm{n}^{\circ}$ 10, pp. 1810-1815, October 1993.

[2] R. A. York, "Nonlinear analysis of phase relationships in quasioptical oscillator arrays", IEEE Transactions on Microwave Theory and Techniques, vol. 41, n¹0, pp. 1799-1809, October 1993.

[3] P. Liao and R. A. York, "Beam Scanning With Coupled VCOs", In Proc. Antennas Propagation Soc. Int. Symp., vol. 2, pp. 836-839, June 1994.

[4] R. A. York and T. Itoh, "Injection and phase-locking techniques for beam control", IEEE Transactions on Microwave Theory and Techniques, vol. 46, pp. 1920-1929, November 1998.

[5] K. Kurokawa, "Injection locking of solid state microwave oscillators", In proc. IEEE, vol. 61, n 10, pp. 1386-1409, October 1973.

[6] R. A. York and P. Liao, "Oscillator Array Dynamics with Broadband NPort Coupling Networks", IEEE Transactions on Microwave Theory and Techniques, vol. 42, n 11, pp. 2040-2045, November 1994.

[7] J. J. Lynch and R. A. York, "Synchronization of Oscillators Coupled Through Narrow-Band Networks," IEEE Transactions on Microwave Theory and Techniques, vol. 49, pp. 237-249, February 2001.

[8] N. Y. Tohmé, J.M. Paillot, D. Cordeau, P. Coirault, "Analysis of the Frequency Locking Region of Coupled Oscillators Applied to 1-D Antenna Arrays," European Microwave Conference, Amsterdam, Holland, pp. 1334-1337, October 2008.

[9] D. Cordeau, JM. Paillot, « Minimum phase noise of an LC oscillator : determination of the optimum operating point of the active part », AEU, International journal of electronics and communications, vol.64, ${ }^{\circ} 9, \mathrm{pp}$. 795-805, september 2010. 